\title{
A simulation study of Texas Hold 'em poker: what Taylor Swift understands and James Bond doesn't
}

\section{Jay Falletta ${ }^{1}$ and Stephen Woodcock ${ }^{1}$}

1. School of Mathematical and Physical Sciences, University of Technology Sydney, Sydney, Australia

Contact: stephen.woodcock@uts.edu.au

ORCiD: 0000-0001-9007-123X (Jay Falletta)

ORCiD: 0000-0003-4903-3164 (Stephen Woodcock)

Keywords: Poker, Simulation, Probability

Mathematics Subject Classification: $91 \mathrm{~A} 60$

\begin{abstract}
:
Recent years have seen a large increase in the popularity of Texas hold 'em poker. It is now the most commonly-played variant of the game, both in casinos and through online platforms. In this paper, we present a simulation study for games of Texas hold 'em with between 2 and 23 players. From these simulations, we estimate the probabilities of each playing having been dealt the winning hand. These probabilities are calculated conditional on both partial information (i.e. the player only having knowledge of his/her cards) and also on fuller information (i.e. the true probabilities of each player winning, given knowledge of the cards dealt to each player.) Where possible, our estimates are compared to exact analytic results and are shown to have converged to 3 significant figures.
\end{abstract}

With these results, we assess the poker strategies described in two recent pieces of popular culture. In comparing the ideas expressed in Taylor Swift's song, New Romantics, and the betting patterns employed by James Bond in the 2006 film, Casino Royale, we conclude that Ms Swift demonstrates a greater understanding of the true probabilities of winning a game of Texas hold 'em poker.

\section{Introduction:}

Over the past two decades, Texas hold 'em poker has seen a huge rise in popularity. It is now the most played variant of the game, both inside and outside of casinos. As of 2016, it is estimated that there are now around 100 million players worldwide [1] Much of this rise has come about as a result of both online poker [2] and the televising of major tournaments.

The game can be played by between 2 and, at least in theory, 23 players. In practice, however, games of more than 10 players are rarely seen. Almost all casinos do not allow more than 10 players in a game. Each player is dealt two cards (hereafter referred to as the hole cards) which are only seen by the player. In addition, there are five cards dealt onto the table (hereafter referred to as the community cards) which are common to all players. Initially the community cards are dealt face down, unseen by all players [3, 4]. A player's final hand consists of five cards which are selected either from his/her hole cards or the community cards. 
In real play, players can choose to withdraw from the game (fold) either after viewing only his/her hole cards, or after three, four or all five community cards have been revealed.

Generally this is done when a player believes he/she has little chance of winning and does not wish to risk further losses backing a losing hand. As such, the winner of a game is not necessarily the player who has the best hand as he/she may have already folded, presumably in the mistaken belief that another player had a superior hand. For the purpose of this mathematical exercise, however, we are not seeking to model or predict how players might choose to act. Instead we are looking at the outcome if all players remain in the game to the end, so that the player with the best hand wins. As such, the work presented here should not be considered as an exercise in attempting to model or predict whether or not a player might win a given game of poker. Rather, this study gives a necessary baseline from which further research into decision theory or human psychology might wish to continue.

The vast majority of recent research interest in Texas hold 'em poker has not been from the mathematical or statistical community, rather from diverse fields such as computer science, behavioural economics, psychology [5] and artificial intelligence. Through exhaustive iteration, the game has been weakly solved and optimal strategies obtained for decision making under certain conditions, including for games with only two players [6, 7]. Similarly, there has been significant progress looking at the effects of irrational and sub-optimal behaviour in poker playing $[8,9]$. Perhaps the most interesting of these look at neuroevolution models, which are trained on opponents' behaviour on prior hands to predict when certain players might be playing suboptimally [10,11]. Impressively, even for a lowdimensional parameter space, some of these models have claimed on average $60 \%$ of table winnings when tested for a two player game [12] In addition to these quantitative studies, a substantial volume of work has been published in popular psychology [3,13], although it remains unclear whether many of the qualitative arguments are supported by quantitative evidence. In this study, we do not consider such models and instead focus solely on the problem of a given player having a winning hand, regardless of whether or not he/she might have already chosen to fold

While non-trivial, the combinatorics needed to calculate the probability of a given player having a certain hand are long-established and widely available through many poker-related websites [14] and popular books. What are less well established, however, are the joint probabilities required to calculate the chance of a given player having a winning hand, given the hole cards he/she is dealt. The combinatorics for these are much more difficult as the hands different players have are not independent. Cards are drawn without replacement from a single deck of 52 cards, so from each player's perspective, his/her opponents' cards are drawn from the 50 remaining cards. Furthermore, depending on which five cards are drawn as the community cards, there can be extremely high correlations between the hands of the different players. In some instances, two players may have hands which have no relevant cards in common. An example of this would be when one player is dealt a pair of Aces and the other is dealt a pair of 2 s. If the community cards were a 3 , a 5 , a 6 , a 7 and an 8 (with four different suits amongst the community cards), then each player's best hand would simply be the hole cards he/she was dealt. At the other extreme, if the community cards happened to form a Royal Flush (i.e. a Straight Flush containing an Ace as its highest card, the best possible hand), then each player's hole cards become irrelevant and all players have the same five cards in common. In this (admittedly unlikely) case, the game ends in a certain tie, however many players there are. 


\begin{tabular}{|c|c|c|}
\hline Hand & Definition & $\begin{array}{c}\text { Number of possible } \\
\text { hands }\end{array}$ \\
\hline Straight Flush & $\begin{array}{c}5 \text { consecutively ranked cards of } \\
\text { the same suit }\end{array}$ & 41,584 \\
\hline 4 of a Kind & 4 cards of the same rank & 224,848 \\
\hline Full House & $\begin{array}{c}\text { 3 cards of the same rank and a } \\
\text { different 2 of the same rank }\end{array}$ & $3,473,184$ \\
\hline Flush & 5 cards of the same suit & $4,047,644$ \\
\hline Straight & $\begin{array}{c}\text { 5 consecutively ranked cards, } \\
\text { not all of the same suit }\end{array}$ & $6,180,020$ \\
\hline 3 of a Kind & 3 cards of the same rank & $6,461,620$ \\
\hline 2 Pairs & $\begin{array}{c}\text { 2 cards of the same rank and a } \\
\text { different 2 of the same rank }\end{array}$ & $31,433,400$ \\
\hline Pair & 2 cards of the same rank & $58,627,800$ \\
\hline High Card & None of the above & $\left(\begin{array}{c}52 \\
7\end{array}\right)=13,294,46$ \\
\hline & Total &
\end{tabular}

Table 1. Definition of poker hands ordered from best to worst. Where multiple players have equal hands, a higher rank within that hand wins. For example, a pair of Kings would beat a pair of 3s.

\section{Methods:}

Simulations were undertaken using the Individual-Based Modelling language Netlogo [15]. Each of the 52 cards of a standard deck was programmed as an individual. For each single card dealt, one of the remaining cards was selected uniformly at random from the deck and removed from the deck for future deals within that game. This ensured that the simulations were of unbiased selections at random and without replacement from a perfectly-shuffled deck.

Initially, validation simulations were run to ensure that the output agreed with known combinatorics results. For games with between 2 and 23 players, 10 million runs of each game were simulated and each player's best hand recorded. This provided a total of 220 million simulated hands. The relative frequency with which each type of hand was observed was then calculated and found to agree with theoretical values for all hand types to within 5 decimal places.

For these runs, only the probability of each player winning the game was recorded. This was again used for validation purposes and showed two key characteristics. Firstly, for a fixed number of players, the proportion of games won by each player was equal. Secondly, the proportion of games won by a player in a game of $N$ players scaled approximately $\sim 1 / N$ (although slightly faster than this, as the rate of ties i.e. games with no outright winner increased monotonically as the number of players increased.) 


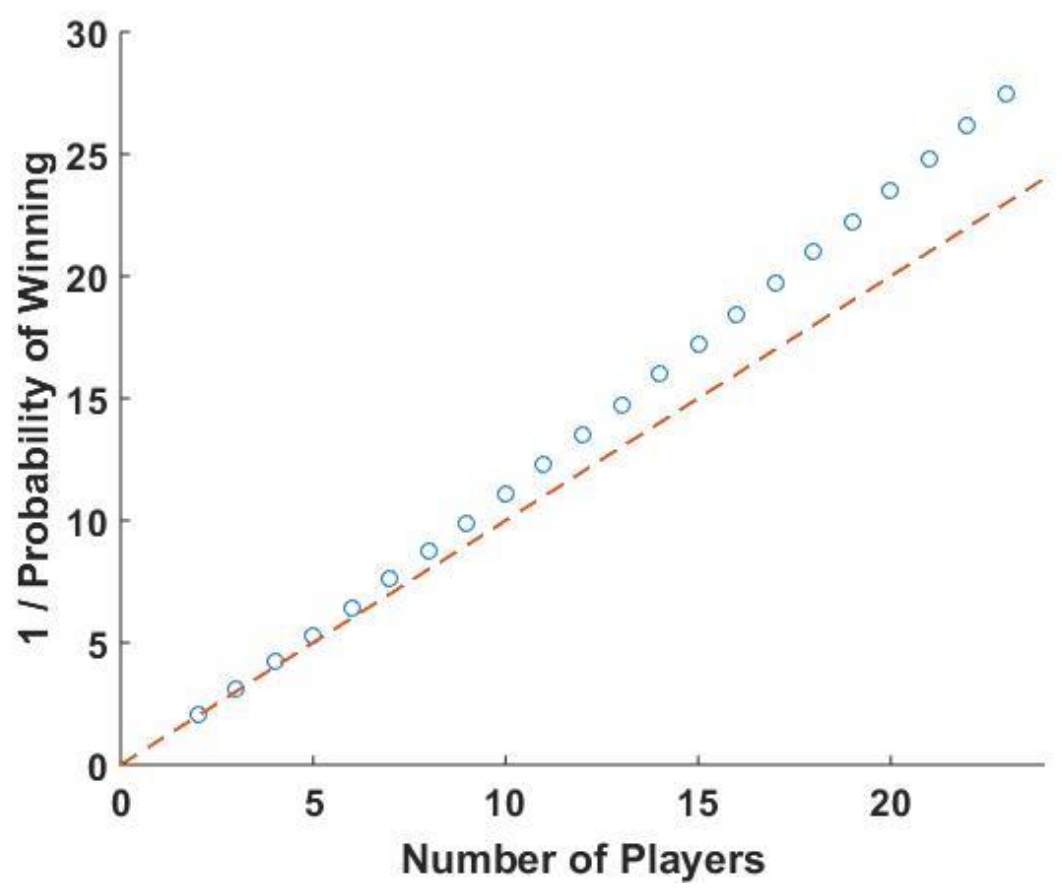

Figure 1. Plot of the reciprocal of the probability of a given player winning the game for different numbers of players. The dashed line $y=x$ is included for comparison.

\section{Numerical Results:}

Neglecting suits, except to note if the two hole cards are of the same or different suits, there are 325 different (ordered) types of hole card combinations. This consists of 169 ordered pairs of cards of different suits plus 156 ordered pairs of cards of the same suit. We retained working with ordered pairs as an additional check of convergence i.e. we sense-checked our estimates by ensuring that the probability of winning with Card A and Card B as hole cards was equal to the chance of winning with Card B and Card $A$.

We then obtained matrices of winning probabilities for all possible hole card combination types and all possible player numbers. Here, we present (Figure 2) only the probabilities for a 10 player game, as these are indicative of the patterns observed for other numbers of players. These probabilities were estimated from 5 million simulations of each of the 325 types of hole card combination (1.625 billion total games in total.)

Two such matrices of probabilities were obtained - one for the case when the two hole cards are of different suits and one for when they are of the same suit. For both of these, the element in the ith row, jth column of the matrix gave the probability of the player winning the game given hole cards numbered $i+1$ and $j+1$ (assuming the Jack, Queen, King and Ace are valued at 11,12,13 and 14 respectively.) All probabilities were estimated to 3 significant figures and we verified that both matrices were symmetric as, for example, two players 
would have the same chance of winning given were dealt a King and a 3 and the other a 3 and a King.

Both Cards Different Suits

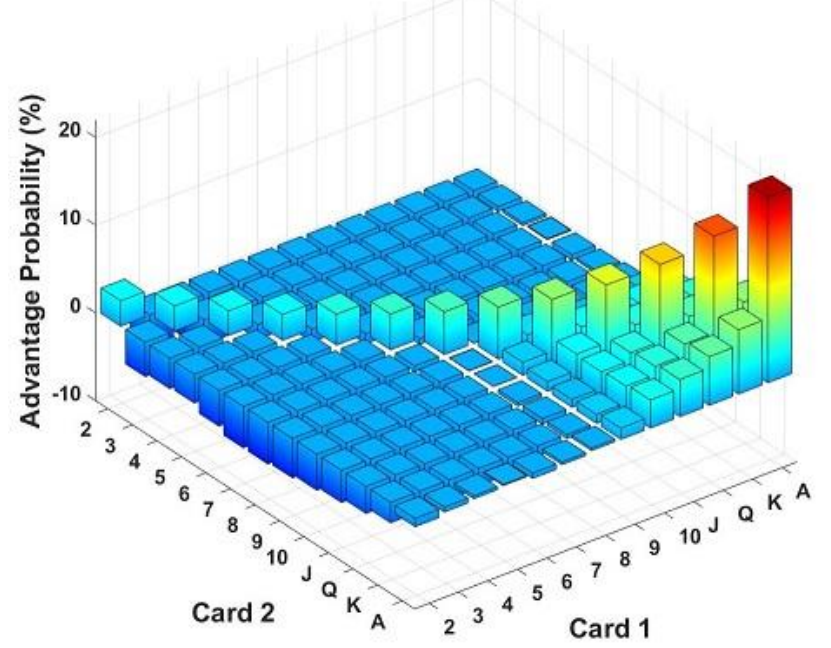

Both Cards Same Suit

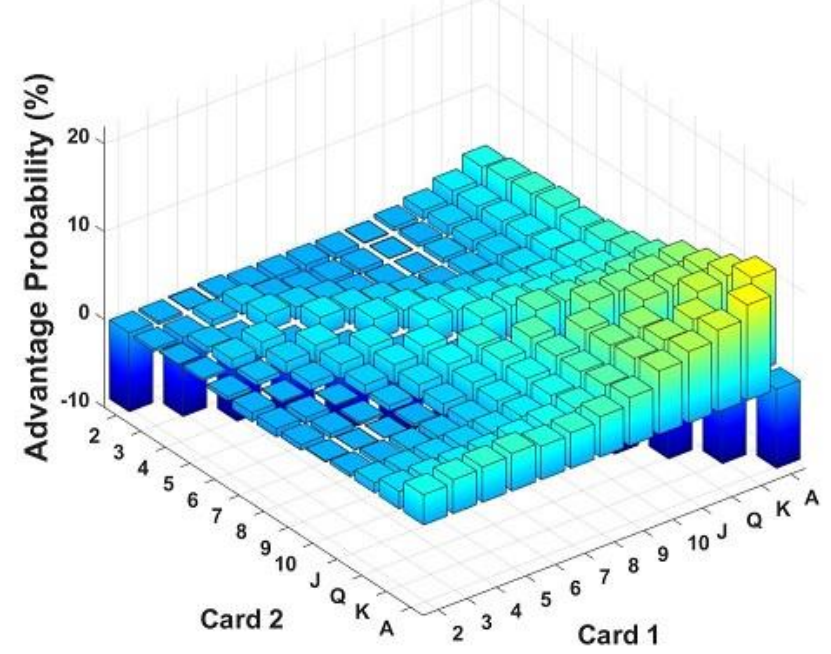

Figure 2. Graphs showing the advantage probabilities of a player winning a 10 player game given knowledge of his/her hole cards (on the two horizontal axes) for: a) two cards of different suits; b) two cards of the same suit.

\section{Case Study 1: Taylor Swift}

Our initial motivation for this study arose from Taylor Swift's 2014 song, New Romantic. In this song, Ms Swift produces the bold claim that "It's poker. He can't see it in my face, but I'm about to play my Ace." We interpreted this claim to represent her belief that she was likely to win a given game of poker because she held an Ace in her hand which other players could not see. While it seemed intuitively obvious that holding at least one Ace would increase a player's chances of winning a given game, we were initially sceptical of her confidence and somewhat disbelieving of the size of the advantage that knowledge of just one card would bring.

The initial problem, however, is not clearly defined as Ms Swift gives no indication of how many other players are involved in her game. While there is no reason to believe she was playing against more than one opponent, there was some ambiguity around this so, for reasons of completeness, we wished to study all possible numbers of players. As such, we examined the effect of having at least one Ace had on a player's probability of winning with possible numbers of players ranging from 2 to 23 .

For each of the 22 possible numbers of players and for each of the 325 types of hole card combination, we ran 1,000,000 simulations ( 7.15 billion games in total)) and from these we estimated 


$$
\mathrm{P}(\text { Win } \mid \text { At least one Ace in Hole Cards })=\frac{\mathrm{P}(\text { Win } \cap \text { At least one Ace in Hole Cards })}{\mathrm{P}(\text { At least one Ace in Hole Cards })} .
$$

The advantage in probability gained from holding at least one Ace was then calculated as

$$
\operatorname{Adv}(\text { Ace })=\frac{P(\text { Win } \mid \text { At least one Ace in Hole Cards })-P(\text { Win })}{P(\text { Win })} .
$$

Surprisingly, the advantage gained by holding an Ace does not vary monotonically with the number of players. For between 2 and 8 players, increasing the number of players increases the proportional advantage of holding an Ace. Increasing the number of players further (up to 17) then decreases the advantage. For 18 or more players, the advantage then increases once more as the number of players grows. As the number of players changes, the advantage of holding an Ace fluctuates approximately following a third-order polynomial with positive leading coefficient.

This is an interesting result and one for which the reasons are not readily apparent. For low player numbers, the chances are still relatively high that few other Aces have been dealt. As the number of players $N$ increases, the baseline comparison probability (i.e. $P(W$ in $)$ ) scales approximately $\sim 1 / N$, hence the advantage above this increases. In the intermediate region, it becomes increasingly likely that some of the other Aces are in other players' hands, diluting the advantage held. In this region, the decrease in advantage with increasing $N$ clearly drops away faster than $\sim 1 / N$, so the probability advantage drops. Lastly, for very large player numbers, it becomes highly likely that all other Aces have been dealt. In such cases, the advantage of holding an Ace varies little with changes in $N$, but the comparison probability continues to scale approximately $\sim 1 / N$, resulting in an increasing advantage again.

Having observed this surprising result, we then looked at the advantage (or disadvantage) associated with any other card rank. Again, the approximately cubic relationship was observed for all card ranks. Fixing at least one card ranked 10 and above (i.e. 10, Jack, Queen, King or Ace) is advantageous and the strength of the advantage changes with the number of players with an approximately third-order polynomial with positive leading coefficient. Fixing a card of a 9 or below is detrimental to a player's chances of winning and the approximate third-order polynomial in the number of players is seen to have a negative leading coefficient. 

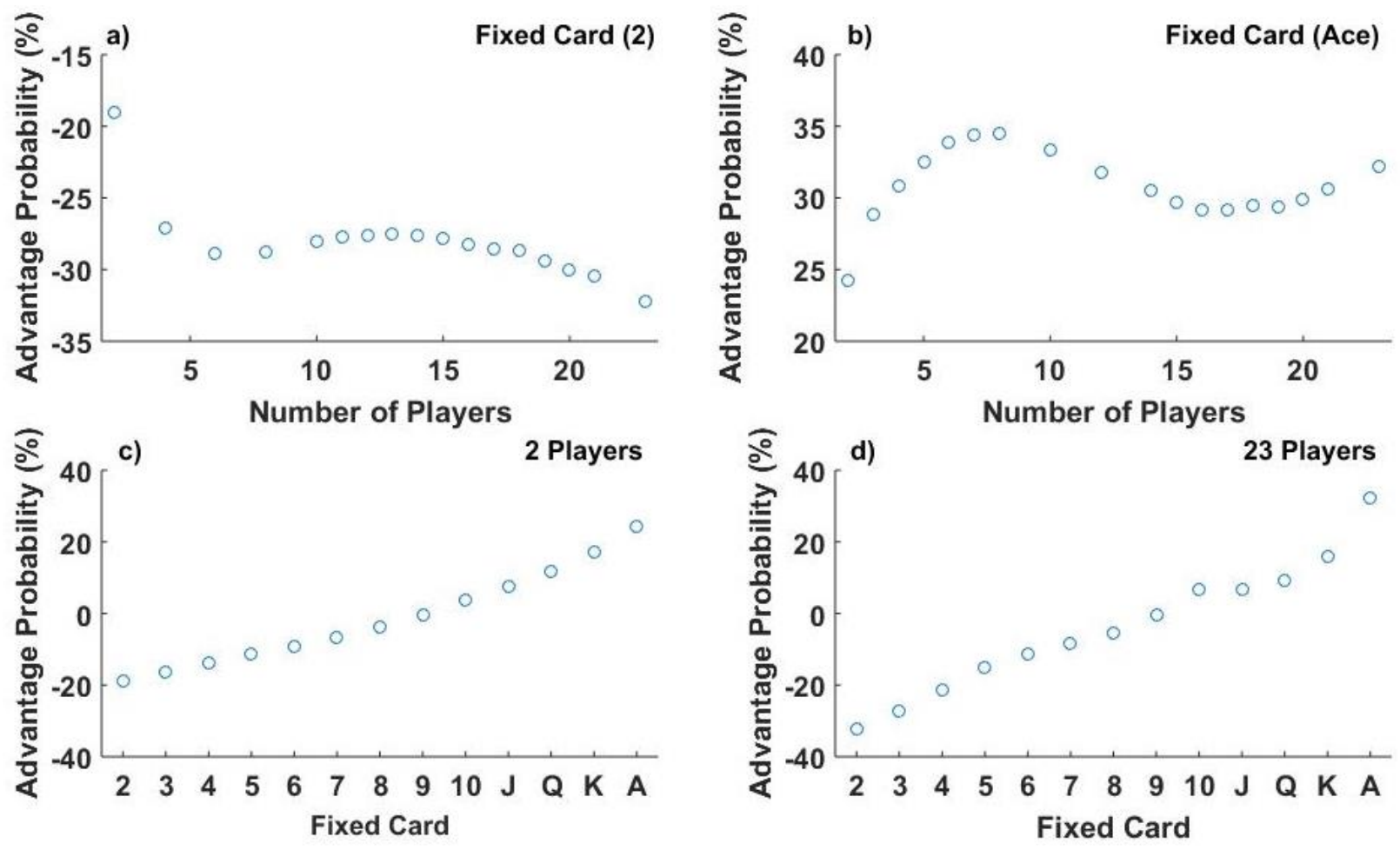

Figure 3. Plots showing the advantage/disadvantage associated with:

a) fixing a 2 in a player's hand for different numbers of players;

b) fixing an Ace in a player's hand for different numbers of players;

c) fixing a different card in a player's hand during a 2 player game;

d) fixing a different card in a player's hand during a 23 player game.

Unsurprisingly, for any number of players, the advantage gained by fixing a given card increases monotonically with the value of the card fixed. In that respect, Taylor Swift's increased confidence is well-founded. It is worth noting, however, that the advantage of holding a high valued card is sufficiently great to overcome the slightly reduced probability of obtaining a Straight, since an Ace can be regarded as the highest card (effectively a 14) or the lowest card (effectively a 1) but not both. For example, a 6 could be any one of five cards in a Straight (i.e. 2, 3, 4, 5, 6 up to $6,7,8,9,10$ ) whereas an Ace can only be either the highest or lowest card in a Straight (i.e. Ace, 2, 3, 4, 5 or 10, Jack, Queen, King, Ace.) A player holding, for example, Queen, King, Ace, 2, 3 would not contain a Straight.

\section{Case Study 2: James Bond}

In the 2006 film, Casino Royale, James Bond is involved in a game of Texas hold'em poker which culminates with four players all competing for a $\$ 115 \mathrm{~m}(\mathrm{USD})$ jackpot. He needs to ensure that he wins this prize to prevent it falling into the hands of Le Chiffre, an alleged mathematical genius who is involved with funding terrorism. The scene culminates in Bond's victory, but we wished to analyse the game as presented to see whether his success was attributable to judicious betting strategies or whether he was simply the beneficiary of good fortune against the odds.

Bond is the last of the four players to call. The first player is dealt a King and a Queen, both of the same suit. The second player has a Pair of $8 \mathrm{~s}$ and the third player (the villain Le Chiffre) has an Ace and a 6 of different suits. Bond's hole cards are a 7 and a 5 of the same suit. We simulated 1,000,000 independent runs of this scenario. 
If Bond were to assess his probability of winning from this point, conditional only on knowledge of his own cards, he would believe himself to have approximately $22.33 \%$ chance of winning. This is lower than his unconditional probability of $23.81 \%$ (i.e. a quarter of the chance that the game does not end tied.) As he is the last player to call and all three previous players have opted to remain in the game (conventionally a sign of their belief that their hands had a good chance of winning), it seems foolish of Bond to even remain in the game. Unbeknown to him, his actual odds of success at this point are even worse than he could know. Conditional on knowledge of all four players' hole cards, his probability of winning is actually a meagre $12.30 \%$.
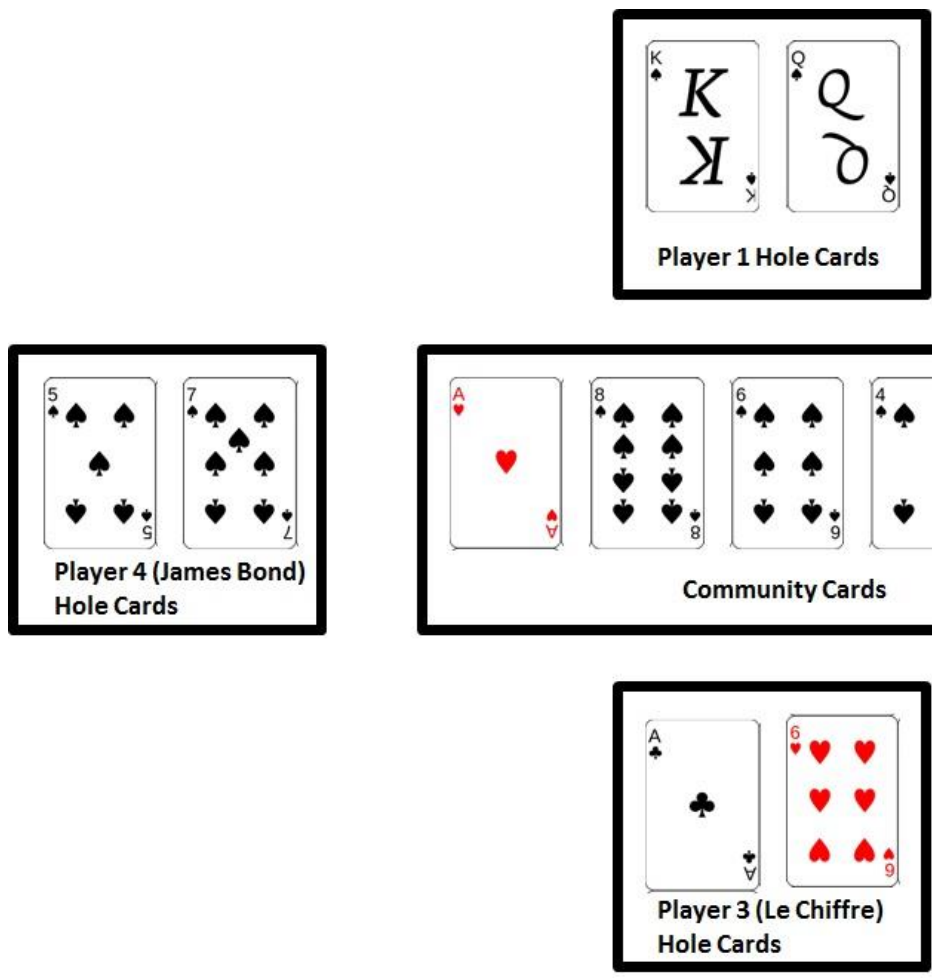

Figure 4. The scenario from the final poker scene in the $2006 \mathrm{film}$, Casino Royale.

Nonetheless, by the time all five community cards have been revealed, Bond emerges triumphantly, but this is far more attributable to an extremely unlikely set of cards being amongst the community cards than of Bond's assessment of the chances he was taking. His success is not even attributable to the poker skill of assessing when other players are bluffing i.e. backing weaker hands in the hope of misleading opponents into folding. Each of the other players did indeed have a superior chance, and hence none was bluffing.

Furthermore, we examined the likelihood of such a scenario arising. In the Casino Royale scene, the weakest final hand is a Flush. From our simulations of four player games, we estimate that, all players having a Flush or better would only arise in less than $0.03 \%$ of games. The chances of a player starting with Bond's hole cards - a 7 and a 5 of different suits - and ending with a Straight Flush or better as he did are even more remote, 
approximately $0.023 \%$. While the scripted scenario may have been cinematically dramatic, such a scene would be exceptionally unlikely to arise by chance alone.

\section{Discussion:}

This study focuses solely on estimating winning probabilities conditional on a player's hole cards. In a real game, a player has several additional chances to either fold and leave the game or else to raise the value of the bet. These opportunities come after three, four and all five of the community cards have been revealed. A more thorough and vastly more computationally expensive study would be needed to assess these probabilities.

In assessing the two popular culture case studies, it is perhaps surprising that the seemingly throwaway remark from Taylor Swift's song conveys a better understanding of probabilities than the seeming expertise of the (ultimately triumphant) James Bond. While she does not give enough information, either about her other hole card or the number of other players, the information of at least a single Ace increases her chances by somewhere between $24 \%$ and $34 \%$, depending on the number of other players.

Interestingly, it is only in the 2006 film Casino Royale that James Bond actually plays Texas hold 'em poker. In both the source material - lan Fleming's 1953 novel of the same name and the earlier (1967) film adaptation, he instead plays baccarat. It is beyond the scope of this study to look at whether Bond's judgement of the game of baccarat is any better than his deeply flawed poker beliefs or whether he is overly-reliant on improbably good luck at all such casino games.

\section{References:}

1. Caballero, J., et al., Cognitive and Performance Enhancing Medication Use to Improve Performance in Poker. Journal of Gambling Studies, 2016. 32(3): p. 835-845.10.1007/s10899015-9576-4

2. Hopley, A.A.B., K. Dempsey, and R. Nicki, Texas Hold'em Online Poker: A Further Examination. International Journal of Mental Health and Addiction, 2012. 10(4): p. 563572.10.1007/s11469-011-9353-2

3. Sklansky, D., The Theory of Poker: A Professional Poker Player Teaches You How To Think Like One. 4th ed. 1999, Las Vegas, NV: Two Plus Two Publishing.978-1880685006

4. van der Kleij, A.A.J., Monte Carlo Tree Search and Opponent Modeling through Player Clustering in no-limit Texas Hold'em Poker, in Artificial Intelligence. 2010, University of Groningen.http://www.ai.rug.nl/ mwiering/Tom van der Kleij Thesis.pdf

5. Meinz, E.J., et al., Roles of domain knowledge and working memory capacity in components of skill in Texas Hold'Em poker. Journal of Applied Research in Memory and Cognition, 2012. 1(1): p. 34-40.https://doi.org/10.1016/j.jarmac.2011.11.001

6. Tammelin, O., et al., Solving heads-up limit Texas holdem., in Proceedings of the 24th International Joint Conference on Artificial Intelligence (IJCAI). 2015

7. Bowling, M., et al., Heads-up limit hold'em poker is solved. Science, 2015. 347(6218): p. 145149.10.1126/science.1259433

8. Javarone, M.A., Is poker a skill game? New insights from statistical physics. EPL, 2015. 110(5): p. 58003.https://doi.org/10.1209/0295-5075/110/58003

9. Javarone, M.A., Modeling Poker Challenges by Evolutionary Game Theory. Games, 2016. 7(4): p. 39.10.3390/g7040039

10. Gilpin, A., T. Sandholm, and T.B. Sørensen, A heads-up no-limit Texas Hold'em poker player: discretized betting models and automatically generated equilibrium-finding programs, in 
Proceedings of the 7th international joint conference on Autonomous agents and multiagent systems - Volume 2. 2008, International Foundation for Autonomous Agents and Multiagent Systems: Estoril, Portugal. p. 911-918

11. Li, R., et al., Opponent's Style Modeling Based on Situations for Bayesian Poker, in Al 2012: Advances in Artificial Intelligence: 25th Australasian Joint Conference, Sydney, Australia, December 4-7, 2012. Proceedings, M. Thielscher and D. Zhang, Editors. 2012, Springer Berlin Heidelberg: Berlin, Heidelberg. p. 385-396.978-3-642-35101-3

12. Lockett, A.J. and R. Miikkulainen, Evolving Opponent Models for Texas Hold 'Em, in IEEE Conference on Computational Intelligence in Games. 2008: Perth, Australia.http://nn.cs.utexas.edu/?lockett:cig08

13. Schoonmaker, A.N., The Pscyhology of Poker. 2000: Two Plus Two.978-1880685259

14. Alspach, B. 7-card poker hands. 2000; Available from: http://people.math.sfu.ca/ alspach/comp20/.

15. Wilensky, U., NetLogo. 1999, Center for Connected Learning and Computer-Based Modeling, Northwestern University.: Evanston, IL.http://ccl.northwestern.edu/netlogo/ 